\title{
Vacuum Phototriodes for the CMS Electromagnetic Calorimeter Endcap
}

\author{
K. W. Bell, R. M. Brown, D. J. A. Cockerill, P. S. Flower, P. R. Hobson, B. W. Kennedy, A. L. Lintern, C. S. Selby,
} O. Sharif, M. Sproston, and J. H. Williams

\begin{abstract}
The measurement of scintillation light from the lead tungstate crystals of the Compact Muon Solenoid (CMS) electromagnetic calorimeter (ECAL) poses a substantial technical challenge, particularly in the endcap regions, where the radiation levels are highest. The photodetectors must be fast, sensitive, radiationhard, and operate with significant internal gain in a magnetic field of 4 Tesla. The measured performance characteristics of the first batches of series production vacuum phototriodes (VPT), developed to satisfy the needs of CMS, will be described.
\end{abstract}

Index Terms-Calorimetry, photodetectors, photomultipliers.

\section{INTRODUCTION}

$\mathbf{T}$ HE Compact Muon Solenoid (CMS) experiment [1] is one of two general-purpose detectors currently being constructed for operation at the LHC proton-proton collider at CERN. The CMS electromagnetic calorimeter (ECAL) detector [2] comprises an array of scintillating lead tungstate crystals; the crystals are slightly tapered to generate a quasipointing geometry, as shown in Fig. 1. The barrel, of length $6 \mathrm{~m}$ and radius $1.75 \mathrm{~m}$, contains approximately 61000 crystals, while the endcaps contain approximately 15000 crystals, each crystal being equipped with a photodetector. The endcap crystals are $30 \times 30$ $\mathrm{mm}^{2}$ in cross section and $220 \mathrm{~mm}$ long, corresponding to a depth of approximately 25 radiation lengths. The calorimeter lies inside the 4-T solenoidal magnetic field of CMS. The benchmark physics channel which drives the ECAL design is the decay of the Higgs boson to two photons; this will be the most significant discovery channel for the Higgs if its mass is less than $130 \mathrm{GeV} / \mathrm{c}^{2}$. The detection of this decay process above background requires that the energy resolution of the ECAL must be less than $1 \%$ for photons of $100 \mathrm{GeV}$ energy.

The experimental conditions expected at the LHC impose stringent demands on the detector technology. The radiation environment during collider operation will be very harsh. The ECAL barrel will receive an integrated gamma dose of up to $4 \mathrm{kGy}$ during 10 years of operation. The dose rate is a strongly increasing function of rapidity, with an expected integrated dose of several tens of $\mathrm{kGy}$ in the central region of the endcap, while the dose at the inner edge of the endcap could be as high as

Manuscript received November 14, 2003; revised March 23, 2004. This work was supported by PPARC.

K. W. Bell, R. M. Brown, D. J. A. Cockerill, P. S. Flower, B. W. Kennedy, A. L. Lintern, M. Sproston, and J. H. Williams are with the CCLRC Rutherford Appleton Laboratory, Chilton, Didcot, Oxfordshire OX11 0QX, U.K.

P. R. Hobson, C. S. Selby, and O. Sharif are with Brunel University, Department of Electronic and Computer Engineering, Uxbridge, Middlesex UB8 3PH, U.K.

Digital Object Identifier 10.1109/TNS.2004.836053

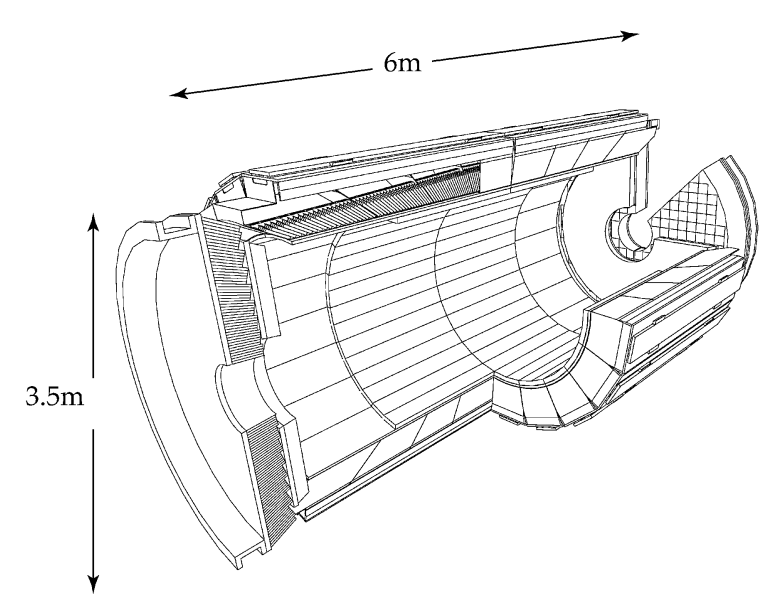

Fig. 1. Schematic view of CMS electromagnetic calorimeter. The vacuum phototriodes will be mounted at the rear of the endcap crystals, seen in the cutaway endcap on the left of the figure.

$200 \mathrm{kGy}$ [3]. The neutron fluence will also be intense, ranging from $10^{13} \mathrm{~cm}^{-2}$ in the ECAL barrel to $10^{15} \mathrm{~cm}^{-2}$ at the inner edge of the endcap. Radiation tolerance is therefore a vital consideration in the detector design.

The LHC will operate at a frequency of $40 \mathrm{MHz}$, so that the interval between beam crossings will be only $25 \mathrm{~ns}$. The ECAL must therefore be capable of responding on this timescale to provide prompt information to the triggering system.

A further constraint on the ECAL photodetector arises from the fact that the scintillation light output of lead tungstate is rather weak; the $\mathrm{Y} / \mathrm{Nb}$-doped crystals used in CMS typically yield 50 photons per $\mathrm{MeV}$ of energy deposited, requiring the use of a photodetector with internal gain.

Finally, the photodetectors must function satisfactorily in the 4-T solenoidal magnetic field of CMS.

The photodetectors chosen for the ECAL barrel are avalanche photodiodes (APDs) [4]. The neutron radiation flux in the endcaps is too severe for these solid-state detectors, and Vacuum Phototriodes (VPTs) will be used. The properties of these devices will be described in the remainder of this paper.

\section{Description of the CMS Vacuum Phototriodes}

A vacuum phototriode (VPT) is a single-stage photomultiplier. A schematic diagram of a VPT is shown in Fig. 2. The anode of the device takes the form of a $10-\mu \mathrm{m}$ pitch mesh of approximately $50 \%$ transparency; electrons liberated from the photocathode are able to pass through the holes in the mesh to strike the dynode, generating secondary electrons which are collected on the anode. 


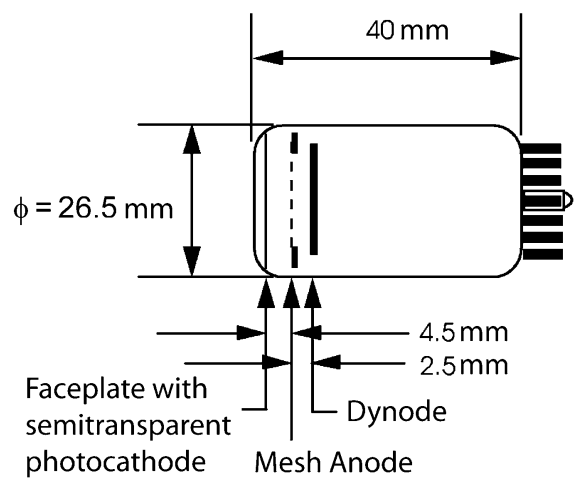

Fig. 2. Diagram of a vacuum phototriode.

The VPTs for the CMS ECAL endcap are manufactured by Research Institute Electron, St. Petersburg, Russia, following a period of development in collaboration with PNPI Gatchina, Russia. A total of 6200 devices have been delivered at the time of writing, comprising a preproduction batch of 500, and 5700 devices manufactured under production conditions. Further information on the development of VPTs for CMS may be found in [5] and [6].

The VPTs delivered so far have a mean quantum efficiency of $22 \%$ at $430 \mathrm{~nm}$, with a standard deviation of $3 \%$, and a mean gain of 10.2 at $0 \mathrm{~T}$, with a standard deviation of 1.8. Each VPT/crystal pair will be individually calibrated, so that these variations do not compromise the resolution of the calorimeter. The specification requires that the loss in output after irradiation to $20 \mathrm{kGy}$ must be less than $10 \%$, while the response in a magnetic field of $4 \mathrm{~T}$, with the VPT axis at $15^{\circ}$ to the field direction, must be at least $75 \%$ of that achieved in zero field.

\section{MEASUREMENTS OF VPT CHARACTERISTICS}

The measured variation in the gain of a typical VPT as a function of the applied voltage is shown in Fig. 3. Two curves are shown, giving the gain as a function of dynode voltage with the anode held at 1000 or $800 \mathrm{~V}$, and the photocathode at ground. The operating point in CMS will be $\mathrm{V}_{\mathrm{a}}=1000 \mathrm{~V}, \mathrm{~V}_{\mathrm{d}}=800 \mathrm{~V}$; at this point, the gain is insensitive to variations in the applied voltage.

All VPTs are tested at the Rutherford Appleton Laboratory (RAL) to determine their response as a function of magnetic field up to $1.8 \mathrm{~T}$, and as a function of angle to the applied field. The automated testing rig at RAL is able to make measurements on 24 VPTs simultaneously, to determine their response to pulses of light. Each VPT is illuminated by a set of four blue LEDs of peak wavelength approximately $420 \mathrm{~nm}$, with the light diffused by a frosted perspex plate to ensure even illumination across the photocathode surface.

A typical set of measurements is shown in Fig. 4. The variation of the VPT output shows a characteristic periodic behavior as a function of angle in a 1.8-T field. When the response is measured as a function of magnetic field, at a fixed angle of $15^{\circ}$, the gain is observed to be constant at fields above $1 \mathrm{~T}$, but variable at lower fields. When the magnetic field is not parallel to the VPT axis, the electrons acquire a transverse drift velocity proportional to the cross-product of the electric and magnetic fields; the maxima and minima seen in Fig. 4 arise because the probability of electron capture on the anode grid varies periodically with the transverse drift velocity. [7].

The VPTs will be installed in CMS at angles between $8^{\circ}$ and $24^{\circ}$ to the magnetic field, and the mean response over this range is taken as a figure of merit characterising the VPT.

Approximately $10 \%$ of the devices, selected at random, are also tested at Brunel University in a 4-T superconducting magnet, at a fixed angle of $15^{\circ}$. These measurements are well correlated with the measurements made at $1.8 \mathrm{~T}$, indicating that the response is stable at high magnetic fields, and that the 1.8-T measurement is a reliable indicator of the VPT performance in CMS.

The measured yield of the VPTs is found to meet the requirements of the CMS ECAL. No systematic variations in the VPT performance have been observed during the period of manufacture.

Detailed measurements of the photocathode response have been made at the University of Split, Croatia, again using blue LED light peaking at $420 \mathrm{~nm}$. The quantum efficiency is found to be uniform over the photocathode area; a typical example is given in Fig. 5, which shows the variation in quantum efficiency in steps of $0.5 \mathrm{~mm}$ across a diameter of the faceplate of a VPT.

\section{RADIATION TOLERANCE}

The high radiation dose expected at LHC implies that the radiation tolerance of the VPTs must be carefully monitored. Tests have shown that the performance of the VPTs is not degraded significantly by neutron irradiation at the levels expected in the central region of the endcap over ten years of LHC running. Gamma irradiation tests have shown that the VPT response is reduced by an amount consistent with the observed loss of faceplate transparency, so that the main area of concern for CMS is the induced absorbance of the faceplate glass expected during the 10-year lifetime of the experiment.

The faceplates of the CMS VPTs are made of a radiation-hard UV-transmitting borosilicate glass, which is manufactured in small batches. Before being used for VPT production, several faceplates from each batch are irradiated in the dark at a temperature of $19^{\circ} \mathrm{C}$ to a dose of $20 \mathrm{kGy}$ over a 48 -h period using a ${ }^{60} \mathrm{Co}$ source at Brunel University. This dose is close to that expected in the central region of the endcap after ten years running in CMS. After irradiation the induced absorbance of the sample is determined as a function of wavelength; a measurement on a typical glass is shown in Fig. 6. The total transmission loss after convolution with the lead tungstate scintillation spectrum, shown as an inset in Fig. 6, must be less than 10\% for the batch to be accepted for use in VPT production.

\section{SUMMARY AND CONCLUSION}

Vacuum Phototriodes have been developed that meet the demanding requirements of the CMS ECAL endcap. Approximately 15000 of these devices are required, of which 6200 have already been delivered by the manufacturer (at mid-October 2003). The performance of all of these VPTs has been measured in a 1.8-T magnetic field; the measurements show that the performance of the VPTs reaches the level required to allow the 


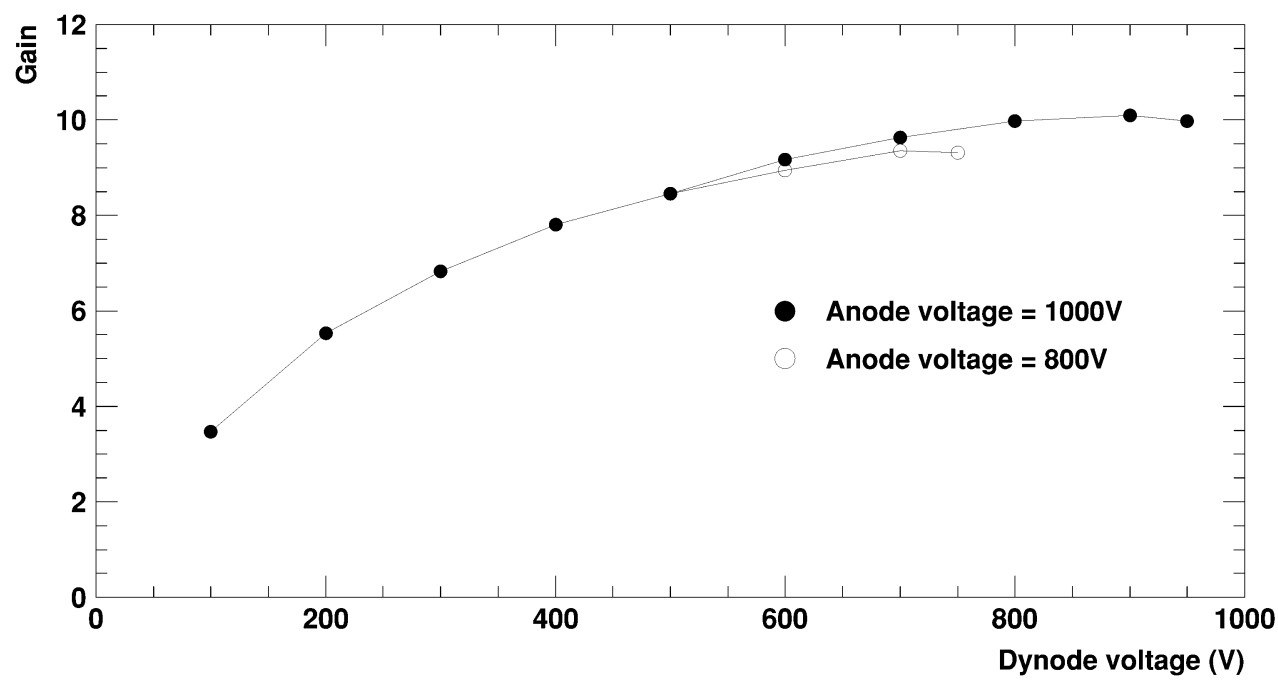

Fig. 3. Variation in gain of typical VPT with applied anode and dynode voltage, at $0 \mathrm{~T}$.

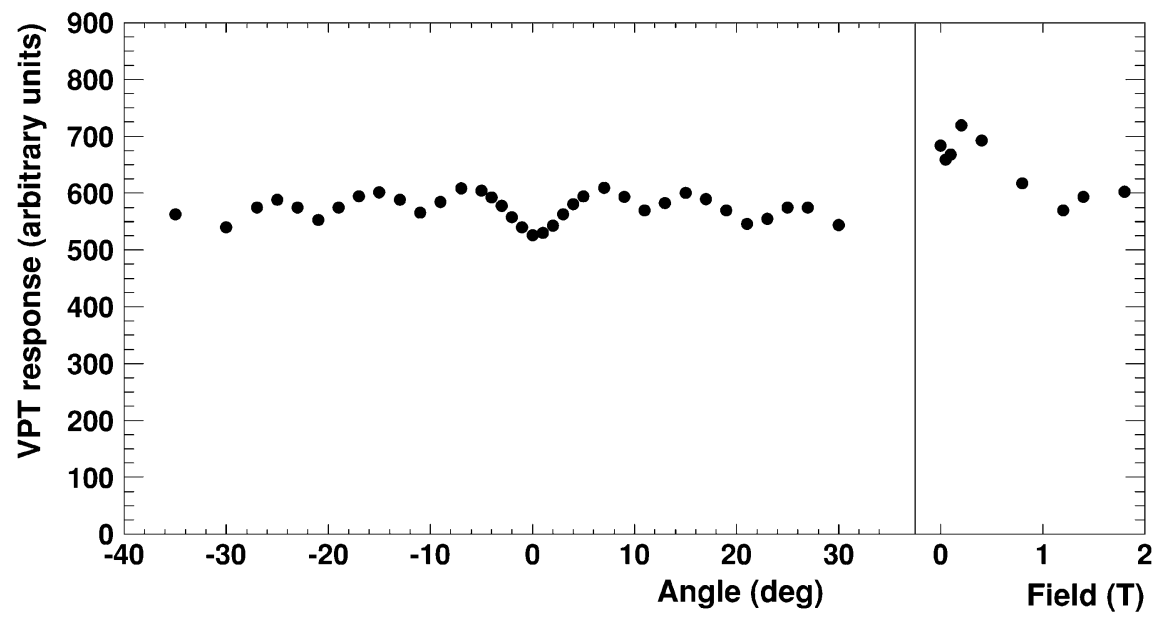

Fig. 4. Response of VPT (arbitrary units) as a function of angle (left-hand side) in a 1.8-T field, and magnetic field (right-hand side) at an angle of $15^{\circ}$.

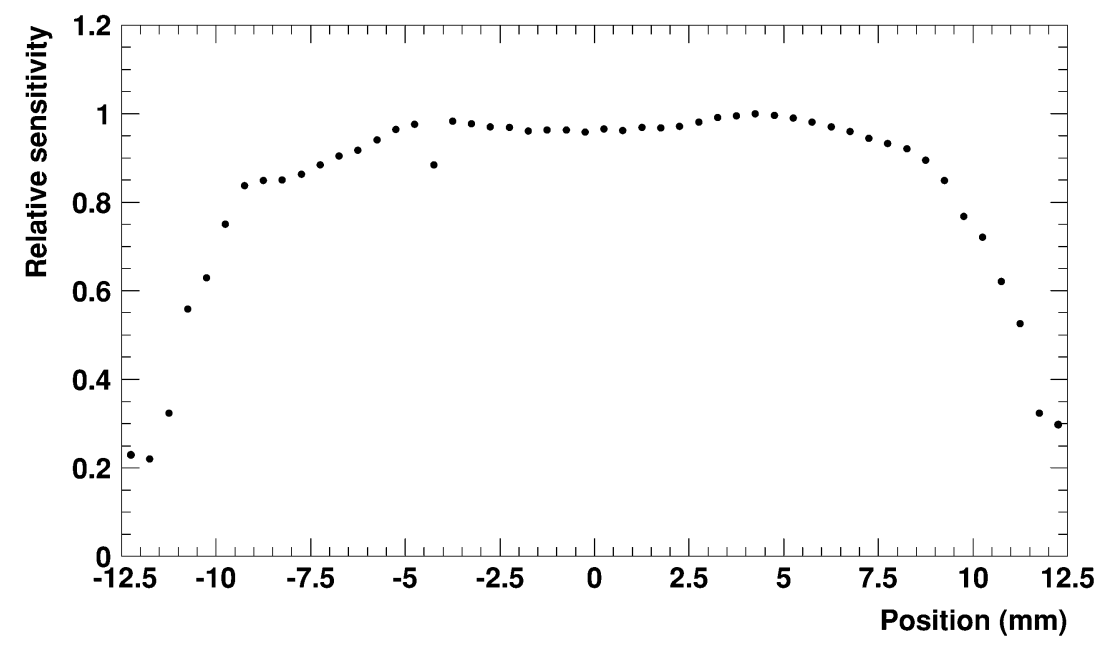

Fig. 5. Variation in relative photocathode efficiency in steps of $0.5 \mathrm{~mm}$ across a diameter of the VPT faceplate.

ECAL endcap detector to achieve an energy resolution of less than $1 \%$ for photons of $100 \mathrm{GeV}$ energy. About $10 \%$ of the devices have also been tested at $4 \mathrm{~T}$; these measurements are found to correlate well with the results obtained at $1.8 \mathrm{~T}$. The radiation tolerance of the VPTs is assured by the irradiation and testing of all faceplate glass batches prior to manufacture. 


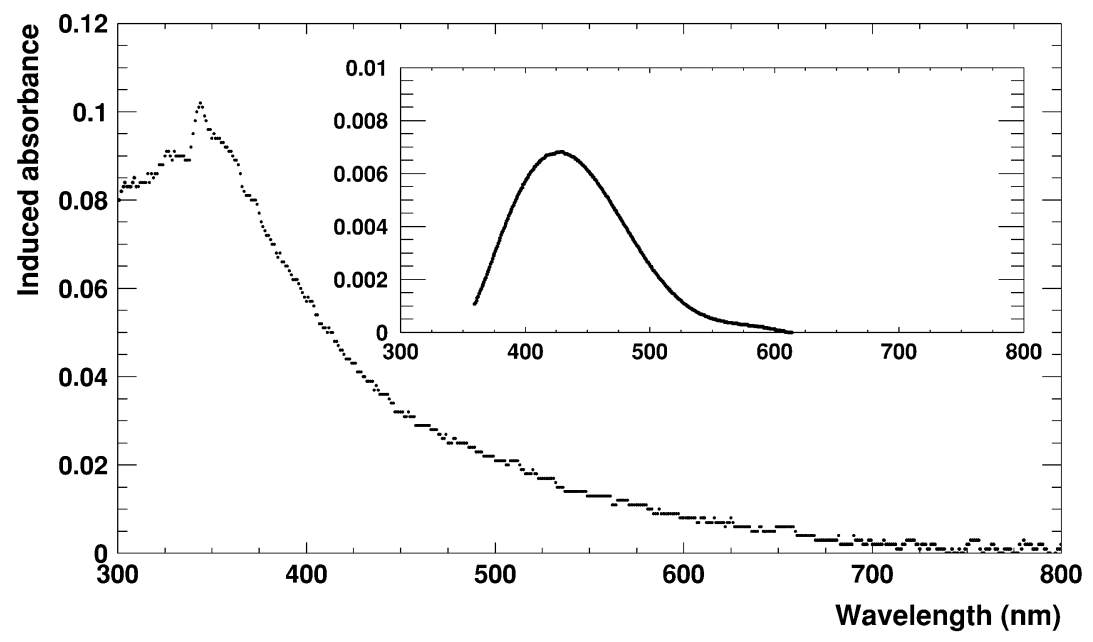

Fig. 6. The induced absorbance of a typical faceplate glass sample, measured after exposure to a gamma dose of $28.2 \mathrm{kGy}$ from a ${ }^{60} \mathrm{Co}$ source. The inset shows the $\mathrm{PbWO}_{4}$ scintillation spectrum (relative output per nm). A convolution of the two curves predicts a reduction in detection efficiency of $\mathrm{PbWO}$ light of $3.1 \%$ for faceplates made of this glass. The structure seen in the absorbance data at wavelengths below $350 \mathrm{~nm}$ is an artefact of the measurement procedure, and lies outside the range of the $\mathrm{PbWO}_{4}$ scintillation spectrum.

\section{ACKNOWLEDGMENT}

The authors would like to acknowledge the work of Research Institute Electron, St. Petersburg, in manufacturing and delivering VPTs according to the CMS technical specifications and the agreed delivery schedule. They also thank their colleagues at PNPI Gatchina for their collaboration in developing the VPT technology and in studying the production devices, and colleagues at the University of Split for their careful measurements of the photocathode response of a number of VPTs.

\section{REFERENCES}

[1] The Compact Muon Solenoid Technical Proposal, The CMS Collaboration, 1994. CERN/LHCC 94-38.
[2] The Electromagnetic Calorimeter Project Technical Design Report, CERN/LHCC 97-33, The CMS Collaboration, Dec. 15, 1997.

[3] M. Huhtinen, "Dose profiles in ECAL crystals for various irradiation conditions," CERN, CMS NOTE 1998/055, Sept. 2, 1998.

[4] K. Deiters et al., "Radiation hard avalanche photodiodes for CMS ECAL," Nucl. Instrum. Methods, vol. A504, pp. 44-47, 2003.

[5] K. W. Bell et al., "The development of vacuum phototriodes for the CMS electromagnetic calorimeter," Nucl. Instrum. Methods, vol. A469, pp. 29-46, 2001.

[6] K. W. Bell et al., "The response to high magnetic fields of the vacuum phototriodes for the compact muon solenoid endcap electromagnetic calorimeter," Nucl. Instrum. Methods, vol. A504, pp. 255-257, 2003.

[7] J. E. Bateman, "The operation of vacuum phototriodes in a non-axial magnetic field—A Monte Carlo study," CERN, CMS NOTE 1999/032, Dec. 21, 1998. 